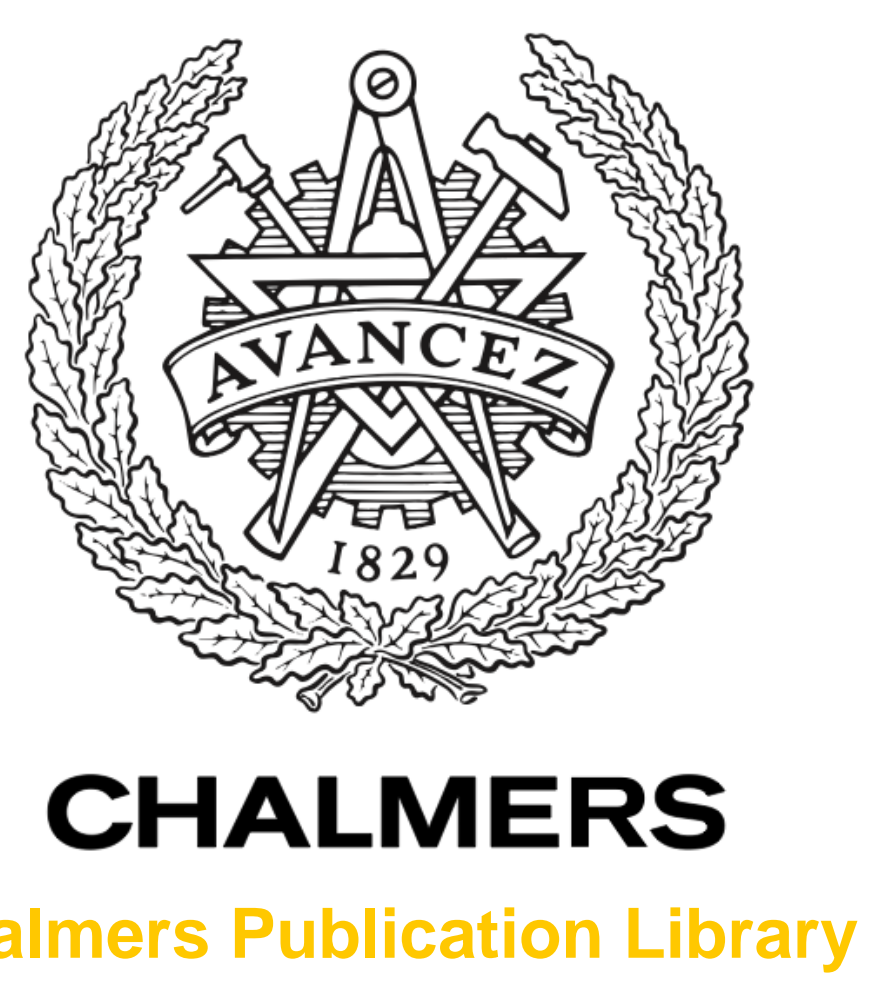

Chalmers Publication Library

\title{
MIMO LTE OTA measurements in reverberation chamber: rich isotropic reference environment makes agreement with theoretical system model
}

This document has been downloaded from Chalmers Publication Library $(\mathrm{CPL})$. It is the author's version of a work that was accepted for publication in:

Proceedings of 6th European Conference on Antennas and Propagation, EuCAP 2012. Prague, 26-30 March 2012

Citation for the published paper:

Kildal, P. ; Orlenius, C. ; Carlberg, U. (2012) "MIMO LTE OTA measurements in reverberation chamber: rich isotropic reference environment makes agreement with theoretical system model". Proceedings of 6th European Conference on Antennas and Propagation, EuCAP 2012. Prague, 26-30 March 2012 pp. 1536-1539.

http://dx.doi.org/10.1109/EuCAP.2012.6206564

Downloaded from: http://publications.lib.chalmers.se/publication/156668

Notice: Changes introduced as a result of publishing processes such as copy-editing and formatting may not be reflected in this document. For a definitive version of this work, please refer to the published source. Please note that access to the published version might require a subscription. 


\title{
MIMO LTE OTA Measurements in Reverberation Chamber: Rich Isotropic Reference Environment Makes Agreement with Theoretical System Model
}

\author{
Per-Simon Kildal ${ }^{1}$, Charlie Orlenius ${ }^{2}$, Ulf Carlberg ${ }^{3}$ \\ Chalmers University of Technology, 41296 Gothenburg, SWEDEN, per-simon.kildal@,chalmers.se \\ Bluetest AB, Götaverksgatan 1,41755 Gothenburg, SWEDEN, charlie.orlenius@bluetest.se \\ SP Technical Research Institute of Sweden, Box 857, 50115 Borås, SWEDEN, ulf.carlberg@sp.se
}

\begin{abstract}
The reverberation chamber has during the last 10 years been developed into an accurate instrument for OTA measurements, having a well-defined rich isotropic multipath environment. This has been introduced as a new reference environment for testing of wireless devices, complementing the traditional anechoic reference environment. The rich isotropic multipath environment and the definition of an ideal errorcorrecting digital receiver have made it possible to develop simple theoretical models that predict the measured throughput data rate in a reverberation chamber versus received power within a few tenth of dBs The present paper overviews the developments of the reverberation chamber that has made it possible to achieve such agreement, as well as the on-going work of determining relevance of the rich isotropic environment compared to real-life environments.
\end{abstract}

\section{INTRODUCTION}

The reverberation chamber has during the last 10 years been developed into such a fast, accurate and cost-effective instrument for emulating rich isotropic multipath and thereby characterizing small antennas and wireless terminals Over-The-Air (OTA) during fading. The principle of operation of the chamber is well understood [1], including that the average transfer function is proportional to total radiation efficiency of the antennas independent of their radiation [2]. It has proven its capability of measuring diversity gain [3], maximum available MIMO capacity [4, 5] of multi-port antenna systems, and total radiated power and receiver sensitivity of wireless devices. The latter can be obtained both as total isotropic sensitivity (TIS) and average fading sensitivity (AFS), the latter measured during continuous fading [6].

Lately, the latter AFS procedure has been extended to measure throughput of complete wireless systems with MIMO capability, such as for WLAN 802.11n, LTE and WiMAX. In this connection it is important that it is possible to change the time and frequency domain characteristics of the chamber (Doppler spread, time delay spread, coherence bandwidth) to emulate different real-life environments [7], so that both frequency-selective and frequency-flat fading can be tested. A new simple theoretical model for throughput of LTE device with MIMO and OFDM frequency diversity has been presented [8], showing perfect agreement with measurements within a few tenths of $\mathrm{dB}$ between the measured and theoretical throughput curves. The paper summarizes how such good agreement is possible.

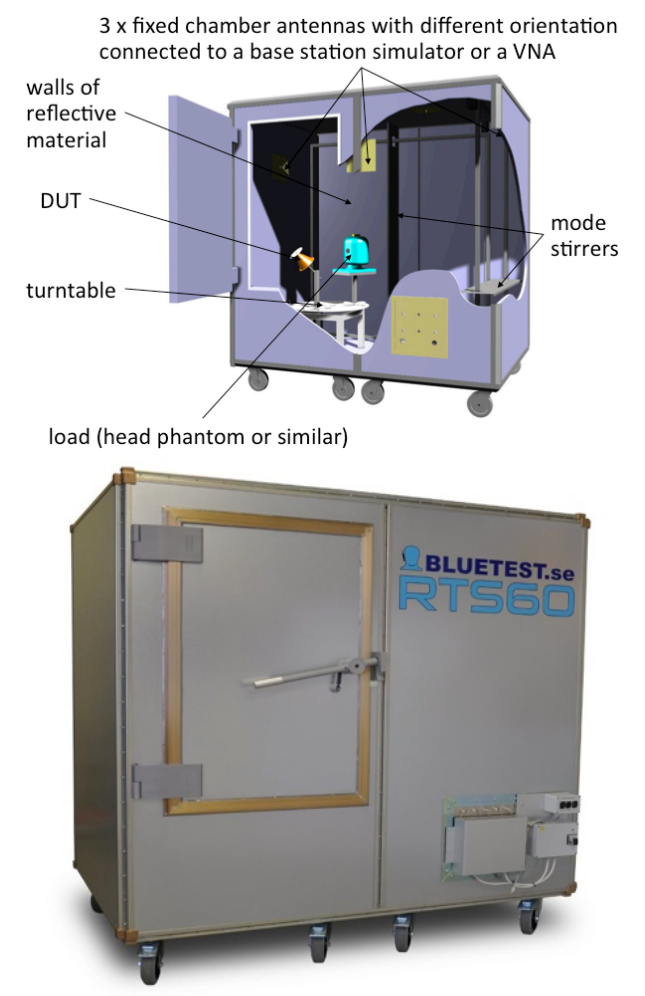

Figure 1. Drawing of typical reverberation chamber with stirrers and chamber antennas (upper) and photo of Bluetest RTS60 chamber.

\section{RICH ISOTROPIC REFERENCE ENVIRONMENT}

Traditionally antennas were designed for pure Line-OfSight (LOS) situations and located on masts and rooftops, and they were characterized in anechoic chambers. The free space anechoic environment is not present in real-life, but still we trust the anechoic reference environment because we are used to it and know the extra guidelines needed for ensuring good LOS performance also in real-life non-free-space situations: We know for instance from textbooks how high a mast must be in order to avoid destructive ground reflections. Modern small antennas for 
wireless systems are on the contrary used everywhere such as in between buildings and indoor. They do not depend on LOS and can effectively handle the statistical field environment and fading due to multipath. Therefore, we have introduced rich isotropic multipath [4] as new reference environment for characterizing such systems (Figure 2). This rich isotropic environment may also not be exactly present in real-life situations, but we claim it is still representative, even though the extra guidelines needed to ensure good real-life performance still remains to be determined.

\begin{tabular}{|c|c|c|c|}
\hline Environment & $\begin{array}{c}\text { Equivalent } \\
\text { measurement } \\
\text { method }\end{array}$ & $\begin{array}{c}\text { Antenna quality } \\
\text { measure }\end{array}$ & $\begin{array}{c}\text { MIMO and } \\
\text { diversity } \\
\text { capability }\end{array}$ \\
\hline $\begin{array}{c}\text { Rich isotropic } \\
\text { multipath }\end{array}$ & $\begin{array}{c}\text { Reverberation } \\
\text { chamber }\end{array}$ & $\begin{array}{c}\text { Total radiation } \\
\text { efficiency }\end{array}$ & Yes \\
\hline $\begin{array}{c}\text { Real-life } \\
\text { environments }\end{array}$ & ?? & $\begin{array}{r}\text { No unique quality } \\
\text { measure }\end{array}$ & Yes \\
\hline $\begin{array}{c}\text { Free space } \\
\text { (LOS) }\end{array}$ & $\begin{array}{c}\text { Anechoic } \\
\text { chamber }\end{array}$ & $\begin{array}{c}\text { Realized gain }= \\
\mathrm{e}_{\text {rad }} \text { * Directivity }\end{array}$ & No \\
\hline
\end{tabular}

Figure 2. Table showing the two extreme reference environments and the real-life environments in between the two extremes.

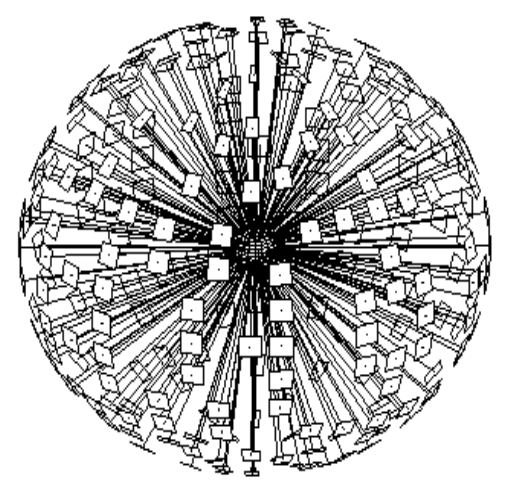

Figure 3. "Ball" of rays of uniformly distributed AoA for illustration of rich isotropic environment [1].

The uniformly distributed ray ball in Figure 3 can illustrate the rich isotropic environment. The angles of arrival (AoA) are uniformly distributed over the unit sphere, and the amplitudes and phases of all rays are randomly distributed in amplitude and phase, creating a rich isotropic environment where the average received power on the antenna (when it is moved around) is independent of the shape of its far field function and depends only on total radiation efficiency. The rich isotropic environment can be emulated in a reverberation chamber. The richness normally means that there are more than 100 waves in the environment.

The anechoic chamber and the rich isotropic environment are two extreme environments that complement each other.

\section{USER-DISTRIBUTED RANDOM LOS}

We need extra guidelines for wireless devices tested in rich isotropic environments, to ensure good performance also in not-so-rich anisotropic environments. We think that a good approach to go ahead with such studies is to concentrate on studying user-distributed random LOS. This can be considered as an extreme not-so-rich environment. We can still consider it as being isotropic if the userdistributed LOS has a completely arbitrary AoA. This will often be the case, in particular for modern tablets that can be used in any orientation. In fact, AoA can also be considered isotropic for handheld phones, as illustrated in Figure 4. Studies are ongoing to determine such guidelines using the in-house computer code ViRM-lab that also is available for others [9]. We can already state that random LOS makes it advantageous to use different polarizations on the terminal and the base station, such as a circularlypolarized base station and a linearly-polarized terminal antenna. Also, if the terminal antenna is directive, the far field functions at all antenna ports together should fill the whole space. Such radiation pattern directional diversity is more important than polarization diversity under random LOS.
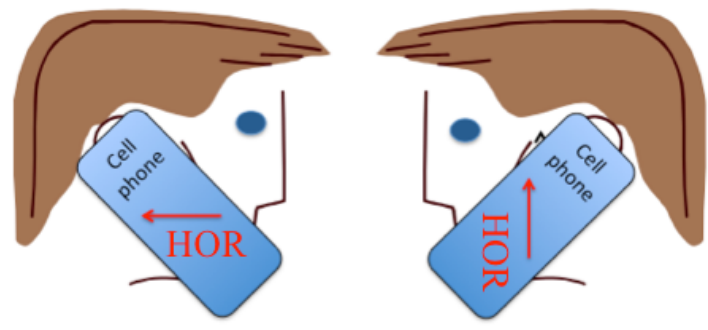

Figure 4. Illustration of the fact that the vertical direction is undefined relative to the geometry of a handheld phone-type terminal. A horizontal arrow and the text HOR is taped on to the phone when this is held on the right side of the head (left drawing). We see that this taped arrow and "HOR" text becomes vertical when the phone is held on the left side of the head (right drawing).

\section{GENERATING FREQUENCY-SELECTIVE FADING IN REVERBERATION CHAMBER}

For testing of the performance in fading environments it is important to be able to emulate fading in the frequency domain that is both frequency-flat and frequency-selective. Thus, we must be able to change the coherence bandwidth. This is possible with the reverberation chamber by introducing different loads in the chamber. Delay-spreads between $30 \mathrm{~ns}$ and $200 \mathrm{~ns}$ are readily available. This is a major factor making good agreement between theoretical and measured data rate throughput in [8]. The average delay spread and coherence bandwidth of the chamber are related as described in [7], where it is also shown that the coherence bandwidth is identical to the average mode bandwidth of the chamber, when they are properly defined. The latter is almost constant with frequency for a given chamber load, and it determines the average power transfer function of the chamber, also being dependent on volume of the chamber and frequency (Hill's formula $[2,10]$ ). 


\section{CALIBRATION AND UNCERTAINTY}

The key behind the success of all measurement methods is thorough calibration and good uncertainty. The basic operation of the reverberation chamber is described by mode stirring, and the associated average power tranfer formula introduced by David Hill [2]. This is based on mode stirring being the only effect of power transfer between two antennas in the chamber, i.e. no LOS components being present. In reality there will be an unstirred component. In [10] we formulate this unstirred component as a LOS component, and by reducing and stirring this LOS we are able to reduce it (referred to as platform stirring and polarization stirring), and we achive uncertainties with STD below $0.2 \mathrm{~dB}$ at high frequency for small loads, see Figure 5. The uncertainty depends on the loads in the chamber, but it is acceptable for practical delay spreads down to $30 \mathrm{~ns}$. The new formulation of uncertainty contains the average $\mathrm{K}$-factor of the chamber [11], and this turns out to be the most characterizing parameter of a chamber in addition to the chamber's physical size and the average mode bandwidth mentioned in Section IV.

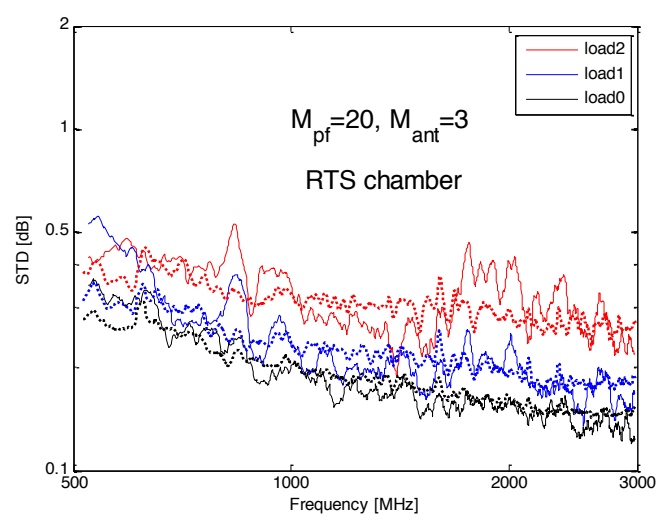

Figure 5. STD in Bluetest RTS reverberation chamber for different loads representing between $200 \mathrm{~ns}$ (load0) and $40 \mathrm{~ns}$ (load2) delay spread [10]. The dotted lines are theoretical based on the formulation using an average $\mathrm{K}$-factor, and the solid lines are measured STDs.

The measured STD curves shown in Figure 5 are based on doing 9 calibration measurements for different positions and orientation of a wideband reference antenna in the chamber. Such wideband calibrations are somewhat timeconsuming, but they are normally not needed. The calibrations used for normal measurements of radiated power, receiver sensitivity of throughput are narrowband and takes normally only minutes.

\section{ThroughPut Model AND MEASUREMENTS}

The recent achievements elated to reverberation chamber measurements are good agreement between measured and theoretical data rate throughput [8]. The theoretical model is based on the fact that digital receivers subject to AWGN channel have a very steep detection threshold, due to the digital error correction codes. For received power levels higher than the threshold all bits are detected, whereas below the threshold nothing is detected.
AWGN means a channel without fading in spatial and frequency domain, achieved by connecting a cable between the base station emulator and the device, this, no fading. We use this property of digital receivers to define an ideal threshold, and based on this the data rate throughput during fading in the reverberation chamber can be very accurately formulated in a simple way. The resulting formula for the data rate throughput is

$$
\text { Tput }=\text { Maxrate } * \operatorname{CCDF}\left(P_{t} / P_{a v}\right)
$$

Here Maxrate is the maximum data rate transmitted by the base station, CCDF is the complementary CDF based on the signal processing implemented in the system, i.e. taking into account all the different diversity combination schemes for MISO, SIMO and OFDM. This CCDF will depend on the embedded efficiencies at the MIMO antenna ports and their correlations, and the frequencyselectiveness of the channels. If we know these factors like in the example Figure 6, we can predict the performance using the formula on a numerically generated Rayleigh fading channel. In addition, we must know the detection threshold $P_{t}$ of the digital receiver, which is readily measured connecting a cable between the receiver and the device, and the maximum available average received power, which we know from the calibration of the average power transfer function and the transmitted power of the instrument. Then, the prediction is in good agreement with measurements as shown in Figure 6 for a $1 \times 2$ SIMO system. We see that we need to include two receive antennas and frequency OFDM diversity in order to explain the measured throughput. The measurement setup is also shown.

The theoretical model is in the process of being upgraded to MIMO multiplexing, and to study the OFDM frequency diversity in more detail.

\section{CONCLUSION}

We have summarized how it is possible to achieve good agreement between measured and theoretical throughput by using a reverberation chamber. The agreement is allocated to the isotropic reference environment, control of the coherence bandwidth (and delay spread) in the chamber, and good calibration and measurement uncertainty. The new ideal threshold receiver model provides under such cases a simple and very accurate prediction and understanding of the measurements.

The good agreement between simple theory and measurements represents an improved quality of OTA measurements, because the lab engineers easily can see if their measurement setup works and what can be the reason for measured performance differences between wireless devices, to ensure correct selection of good devices from bad ones.

The agreement with theory opens also up for testing of advanced system algorithms in practical implementations, e.g. together with more general hardware such as commercial software based radio modules such as GNU radio. 
We have also discussed the relevance of the rich isotropic reference environment compared to real-life environments. Work is ongoing to determine additional guidelines to ensure that devices tested in rich isotropic environments also work well in real-life not-so-rich and anisotropic environments. This study is initially focussed on studying user-distributed random LOS.

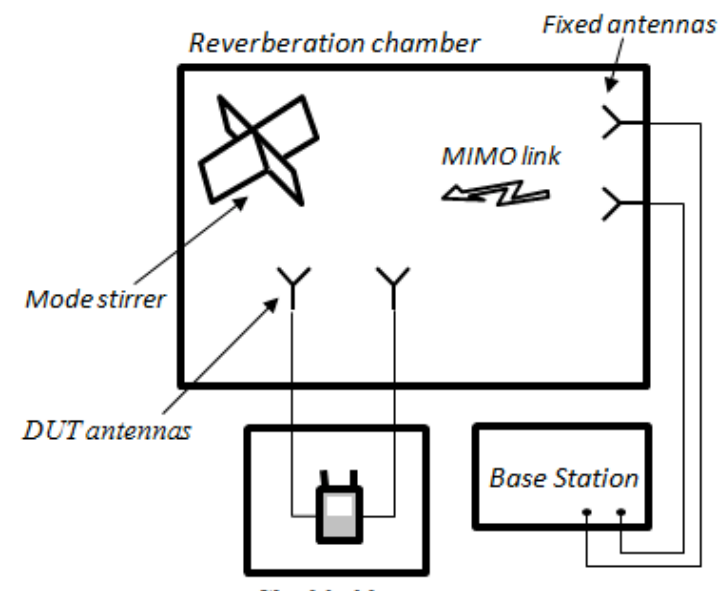

Shielded box

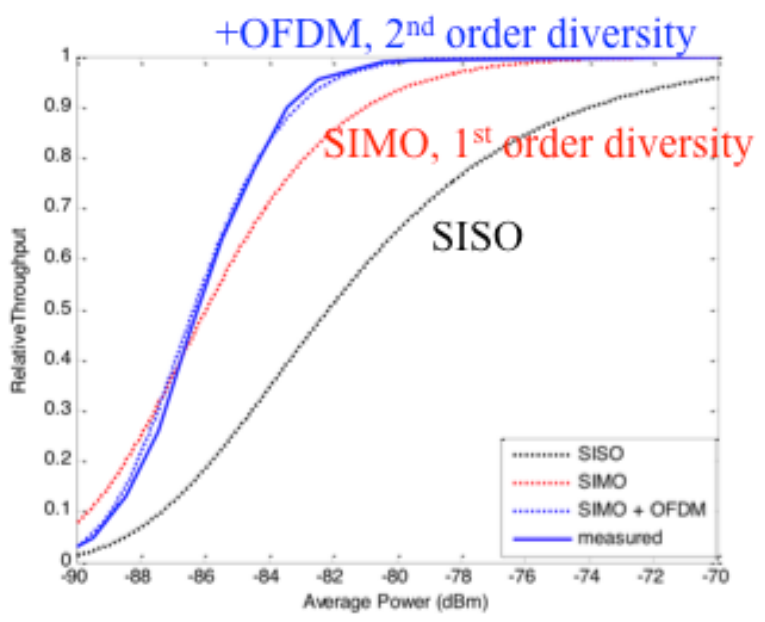

Figure 6. Measurement setup for data rate throughput measurements using low correlation separate antennas in reverberation chamber for $1 \times 2$ SIMO system and comparison between measured (solid line) and theoretical (dotted lines) throughput. The different dotted lines show the effects of single- (SISO, black curve) and dual (SIMO) receive antennas and the additional $2^{\text {nd }}$ order diversity achieved by frequency selective fading and the OFDM.

\section{REFERENCES}

[1] K. Rosengren and P. S. Kildal, "Study of distributions of modes and plane waves in reverberation chambers for the characterization of antennas in a multipath environment," Microwave and Optical Technology Letters, vol. 30, pp. 386-91, 2001.

[2] D. A. Hill, M. T. Ma, A. R. Ondrejka, B. F. Riddle, M. L. Crawford, and R. T. Johnk, "Aperture excitation of electrically large, lossy cavities," Electromagnetic Compatibility, IEEE Transactions on, vol. 36, pp. 169-178, 1994.

[3] P. S. Kildal, K. Rosengren, B. Joonho, and L. Juhyung, "Definition of effective diversity gain and how to measure it in a reverberation chamber," Microwave and Optical Technology Letters, vol. 34, pp. 56-9, 2002.

[4] P. S. Kildal and K. Rosengren, "Correlation and capacity of MIMO systems and mutual coupling, radiation efficiency, and diversity gain of their antennas: simulations and measurements in a reverberation chamber," Communications Magazine, IEEE, vol. 42, pp. 104-112, 2004.

[5] K. Rosengren and P. S. Kildal, "Radiation efficiency, correlation, diversity gain and capacity of a six-monopole antenna array for a MIMO system: theory, simulation and measurement in reverberation chamber," Microwaves, Antennas and Propagation, IEE Proceedings -, vol. 152, pp. 7-16, 2005.

[6] C. Orlenius, P. S. Kildal, and G. Poilasne, "Measurements of total isotropic sensitivity and average fading sensitivity of CDMA phones in reverberation chamber," in 2005 IEEE Antennas and Propagation Society International Symposium, 3-8 July 2005, Piscataway, NJ, USA, 2005, pp. 409-12.

[7] X. Chen, P. S. Kildal, C. Orlenius, and J. Carlsson, "Channel Sounding of Loaded Reverberation Chamber for Over-the-Air Testing of Wireless Devices: Coherence Bandwidth Versus Average Mode Bandwidth and Delay Spread," Antennas and Wireless Propagation Letters, IEEE, vol. 8, pp. 678-681, 2009.

[8] P. S. Kildal, A. Hussain, X. Chen, C. Orlenius, A. Skårbratt, J. Åsberg, T. Svensson, and T. Eriksson, "Threshold Receiver Model for Throughput of Wireless Devices with MIMO and Frequency Diversity Measured in Reverberation Chamber," IEEE Antennas and Propagation Wireless Letters, vol. 10, pp. 1201-1204, October 2011.

[9] U. Carlberg, J. Carlsson, A. Hussain, and P. S. Kildal, "Ray based multipath simulation tool for studying convergence and estimating ergodic capacity and diversity gain for antennas with given far-field functions," in 2010 20th International Conference on Applied Electromagnetics and Communications (ICECom), 20-23 Sept. 2010, Dubrovnik, Kroatia, 2010, p. 4 pp.

[10]P.-S. Kildal, X. Chen, C. Orlenius, M. Franzén, and C. Lötbäck Patané, "Characterization of Reverberation Chambers for OTA Measurements of Wireless Devices: Formulation of Channel Matrix and Uncertainty," submitted to IEEE Transactions on Antennas and Propagation, September 2011.

[11]X. Chen, P. S. Kildal, and S. H. Lai, "Estimation of Average Rician K-Factor and Average Mode Bandwidth in Loaded Reverberation Chamber," Antennas and Wireless Propagation Letters, IEEE, vol. 10, pp. 1437-1440, 2011. 